\title{
Barreiras para comunicação eficaz em saúde
}

\author{
Barriers to effective health communication \\ Barreras para una comunicación eficaz de salud
}

\section{Resumo}

Introdução: a comunicação é fundamental para que as relações interprofissionais se estabeleçam; a forma como se efetiva pode gerar erros e resultar em danos aos pacientes. Objetivo: avaliar os fatores que influenciam as relações humanas e organizacionais em hospitais, capazes de impactar a comunicação da equipe de saúde e implicar na ocorrência dos eventos adversos em dois Hospitais da região metropolitana de Porto Alegre/RS. Metodologia: realizado um estudo de caso múltiplo e incorporado. A coleta de dados entre julho a agosto de 2020, por meio de entrevista semiestruturada, virtual e gravada; para análise dos dados adotou-se o método de análise de Laurence Bardin. Resultados: os resultados estão vinculados às barreiras para uma comunicação eficaz entre equipe de trabalho, as citadas frequentemente foram: gestão e processos, pessoal, relações de poder e carga de trabalho, a maior diferença entre as unidades de análise ocorreu na primeira barreira citada. Conclusão: as dificuldades relacionadas à gestão, processo e relações de poder reforçaram a necessidade de implantação e acompanhamento de protocolos e rotinas, e, a construção de relacionamentos entre equipe baseados no diálogo, pontos frágeis à mitigação de eventos adversos associados à comunicação.

Palavras-chave: Comunicação; Barreiras de comunicação; Segurança do paciente; Erros médicos.

\begin{abstract}
Introduction: communication is essential to establish inter-professional relationships, the way it is effective can generate errors and result in harm to patients. Objective: evaluate the factors that influence human and organizational relationships, being able to impact the communication of the teamwork and implicating the occurrence of adverse events in two hospitals in the metropolitan region of Porto Alegre/RS. Methodology: It was carried out a multiple and embedded case study and data collection between July and August 2020, through semi-structured, virtual and recorded interviews, to analyze the data, Laurence Bardin's method of analysis has been adopted. Results: the results are related to the barriers for effective communication between the teamwork, the ones frequently mentioned were: management and processes, personnel, power relations and workload, the biggest difference between the units of analysis occurred in the first mentioned barrier. Conclusion: the difficulties related to management and process, and power relations, reinforced the need to implement and monitor protocols and routines, and, the construction of relationships between teams based on dialogue, weak points tomitigate adverse events associated with communication.
\end{abstract}

Keywords: Communication; Communication barriers; Patient safety; Medical errors. 


\begin{abstract}
Resumen
Introducción: la comunicación es fundamental para que se den las relaciones interprofesionales, la forma en que es efectiva puede generar errores y daños a los pacientes. Objetivo: evaluar los factores que influyen en las relaciones humanas y organizacionales, capaces de impactar la comunicación del equipo de trabajo e implicar la ocurrencia de eventos adversos en dos hospitales de la región metropolitana de Porto Alegre/RS. Metodología: se realizó un estudio de caso múltiple e incrustado, recolección de datos entre julio y agosto de 2020, mediante entrevistas semi-estructuradas, virtuales y grabadas, para analizar los datos, se adoptó el método de análisis de Laurence Bardin. Resultados: los resultados están vinculados a las barreras para la comunicación efectiva entre el equipo de trabajo, las frecuentemente mencionadas fueron: gestión y procesos, personal, relaciones de poder y carga de trabajo, la mayor diferencia entre las unidades de análisis ocurrió en la primera barrera mencionada. Conclusión: las dificultades relacionadas con la gestión y el proceso, y las relaciones de poder, reforzaron la necesidad de implementar y monitorear protocolos y rutinas, y, la construcción de relaciones entre equipos basadas en el diálogo, puntos débiles para mitigar los eventos adversos asociados a la comunicación.
\end{abstract}

Palabras clave: Comunicación; Barreras de comunicación; Seguridad del paciente; Errores médicos.

\title{
1. Introdução
}

Segurança do paciente e a qualidade assistencial têm ganhado ênfase nos últimos anos, atingindo magnitude internacional. Com isso, agravos e danos aos pacientes relacionados a problemas durante a prestação de serviços em saúde têm mostrado elevada frequência e gravidade, sendo considerado evento adverso (EA), todo incidente que causa danos ao paciente, ou seja, resulta em comprometimento de estrutura ou função do corpo podendo ser físico, social ou psicológico (Brasil Ministério da saúde, 2013).

Desde 2014, em âmbito nacional, todo EA relacionado à assistência na saúde deve ser registrado obrigatoriamente no Sistema de Notificação para a Vigilância Sanitária (SNVS). Utilizando essa base de dados, uma pesquisa revelou, no período de junho de 2014 a junho de 2016, um total de 63.933 notificações, destas, provieram, 96,9\% de hospitais e 0,6\% mortes decorrentes de EA. Vários fatores representaram limites a esta pesquisa, tais como: problemas de sistema, predominância de hospitais como notificadores, escassez de dados sobre as investigações, somadas à baixa oportunidade dos registros (Maia et al., 2018). Diversos trabalhos têm mostrado a associação entre a ocorrência de EAs em hospitais e óbito (Agency for Healthcare Research and Quality, 2017; Cooper, 2017; Pelzang \& Hutchinson, 2018).

Outros desfechos, além de óbito, são provenientes dos eventos adversos, entre eles os desencadeados por falhas no processo de medicação (Hammoudi et al., 2018; Heneka et al., 2019; Manias et al., 2019). Uma revisão integrativa abordou erros de medicação em instituições hospitalares, os mais citados foram: dose imprópria 13,3\%, omissão de dose 11,5\% e horário errado $11,1 \%$. As causas estavam relacionadas ao fator humano, sistema e comunicação, esta última foi entendida pelos pesquisadores como falhas na comunicação verbal ou escrita, bem como erros de interpretação da prescrição. No tocante aos participantes $52 \%$ foram realizados com enfermeiro e/ou equipe de enfermagem (Figueiredo et al., 2018).

Portanto, não há como falar sobre qualidade em saúde e segurança do paciente sem referir-se à comunicação entre profissionais que são responsáveis pelo cuidado. Ao passo que pesquisas apontam a comunicação interpessoal deficiente ou fragmentada, como um dos fatores contribuintes ou uma das principais causas de erros e, consequentemente, de eventos adversos (Brasil Ministério da saúde, 2013; Codier \& Codier, 2005; Green et al., 2017; Heneka et al., 2019; Kahriman \& Öztürk, 2016; Lee \& Doran, 2017; Suliburk et al., 2019; Topcu et al., 2017).

Destaca-se a força de trabalho do enfermeiro que permanece durante maior período com o paciente frente às ações de cuidado. Uma análise explorou o papel histórico e contemporâneo do enfermeiro na promoção da segurança do paciente, entre 1900 a 2015, constatando que a ênfase na segurança do paciente aumentou concomitante à complexidade do atendimento. Neste contexto, os enfermeiros desenvolviam uma identidade profissional, focando, muitas vezes, nas preocupações e soluções de segurança (Kowalski, Sonya L. MSN, RN, ACNS-BC; Anthony, Maureen PhD, RN CE, AJN, n.d.). 
Research, Society and Development, v. 10, n. 7, e10810716218, 2021

(CC BY 4.0) | ISSN 2525-3409 | DOI: http://dx.doi.org/10.33448/rsd-v10i7.16218

Contudo, a criação de um ambiente seguro não é tarefa fácil, depende de muitos fatores e pessoas engrenadas dentro de uma organização, e a comunicação é uma das ferramentas fundamentais para a construção das relações sociais e institucionais que sustentarão a prestação de serviço em saúde de qualidade e um ambiente de trabalho saudável. Frente a isso e considerando o conhecimento e envolvimento do enfermeiro com a segurança do paciente, o objetivo desta pesquisa foi avaliar os fatores que influenciam as relações humanas e organizacionais, capazes de impactar a comunicação da equipe de saúde e implicar na ocorrência dos eventos adversos em dois Hospitais da região metropolitana de Porto Alegre/RS.

\section{Metodologia}

Realizou-se um estudo de caso múltiplo incorporado, a coleta de dados ocorreu no período de julho de 2020 a agosto de 2020 em dois Hospitais de Porto Alegre/RS, um público e outro privado, sendo apenas o último com certificação internacional de qualidade em saúde e/ou segurança do paciente desde 2002.

Participaram da pesquisa 23 enfermeiros assistenciais da emergência e unidades de internação, atendendo ao seguinte critério de inclusão: atuar na assistência direta ao paciente há pelo menos dois anos na instituição, tempo mínimo e razoável para um funcionário participar de treinamentos obrigatórios, adaptação a rotinas, normas e cultura organizacional; referente aos critérios de exclusão: enfermeiros com históricos de afastamento nos últimos dois meses prévios ao período da coleta de dados, por acreditar que isso podia contribuir com o viés de memória, já que as informações foram baseadas em histórias de eventos retrospectivos. A replicação abrangeu duas unidades de análise (hospital privado acreditado e Hospital público sem acreditação), composta por seis enfermeiros de cada departamento (recrutados de forma aleatória e por conveniência na emergência e unidade de internação).

Para início da coleta de dados, garantiu-se as aprovações dos Comitês de Ética em Pesquisa da Universidade Luterana do Brasil (ULBRA), Hospitais coparticipantes, e mediante assinatura do Termo de Consentimento Livre e Esclarecido (TCLE). Utilizou-se entrevista semiestruturada, virtual e gravada, com enfermeiros das distintas unidades e organizações. O instrumento de coleta de dados foi composto por duas partes: a primeira com 8 perguntas fechadas com o objetivo de levantamento do perfil dos participantes e a segunda tratou-se de 7 questões abertas. Para formulação do instrumento foi consultado o questionário da Agency for Healthcare Research and Quality (AHRQ), destinado a avaliar a cultura de segurança de hospitais, por ser validado e implementado por centenas de hospitais nos Estados Unidos e internacionalmente, desde 2004 (AHRQ. Agency for Healthcare Research and Quality., 2004), e também um roteiro de entrevista utilizado em artigo que investigou as barreiras e facilitadores da comunicação interprofissional (Olde Bekkink et al., 2018).

Para análise dos dados foi adotado o método de análise de Bardin (2016). A codificação das unidades de análise e subunidades para a tabulação dos dados foi a seguinte: (H@- hospital privado certificado; H- hospital público sem certificação; E- emergência; UI- unidade de internação), quanto aos participantes da pesquisa o código utilizado foi P, mais a numeração de 1 a 24 correspondente à ordem em que as entrevistas ocorreram.

As categorias referentes às barreiras para uma comunicação eficaz estiveram presentes em todas as questões do instrumento de coleta de dados, sendo estratificadas de maneira geral, ou seja, a frequência de aparição nas diferentes fases do instrumento. Por conseguinte, separou-se a frequência de acordo com as unidades de comparação (H e HC, UI e E). Para a análise de coocorrências, calculou-se a mediana do total das categorias (20,5), e a partir daí criou-se níveis de ocorrência (presente $\leq 10$; baixo: 11 a 19; mediano: 20 a 30; alto: 31 a 40; e alerta $\geq 40$ ), a fim de realçar as associações e dissociações entre as unidades e subunidades de análise.

Para análise de dados e reflexão dos achados realizou-se o aprofundamento e comparação embasados nas mais recentes evidências científicas sobre segurança do paciente, eventos adversos e comunicação, a nível nacional e internacional. 


\section{Resultados}

O perfil dos participantes da pesquisa se caracterizou por profissionais experientes: 17 com 10 anos ou mais na função de enfermeiro, 4, entre 5 a 9 anos, e apenas 2 deles, 4 e 3 anos; entretanto, estes dois menos experientes, já possuíam vínculo empregatício com a instituição, respectivamente, há 9 e 25 anos. Portanto, todos possuíam vivência hospitalar e a ambientação à cultura organizacional. A faixa etária predominante foi entre 30 e 40 anos (n=14); na sequência, acima de 40 anos (n=7); e 2 enfermeiros, 28 e 29 anos.

Quanto aos demais aspectos avaliados, 17 participantes eram do diurno e 6 do noturno; 19, sexo feminino e 4 masculinos; 22 com pós-graduação (17 em nível de especialização, 4, mestrado, e 1 doutorado) e um, somente graduação.

A comunicação foi influenciada pelas relações interpessoais e organizacionais entre a equipe de trabalho, neste contexto os enfermeiros identificaram as barreiras relacionadas a este processo, estas, foram categorizadas em níveis de frequência de aparição nos seus discursos. As dificuldades relatadas, divergiram e convergiram entre as unidades e subunidades de análise, e as categorias relacionaram-se entre si, gerando os erros praticados pela equipe. Estas, podem ser visualizadas nos gráficos abaixo (Gráficos 1 e 2). Conforme a frequência de aparição geral (Gráfico 2), as categorias frequentemente mencionadas, por isso, apresentam-se em níveis de alerta, foram: gestão e processos, pessoal e relações de poder; na sequência, a carga de trabalho, a única na faixa entre 31 a 40 citações, portanto, em nível alto; as categorias de nível mediano, em ordem crescente, encontram-se as barreiras: ambiente, experiência e ação comunicativa; representando o nível baixo, aquelas entre 10 e 19 citações, estão: relações entre equipe, atenção, conhecimento, liderança e convivência; já as categorias presentes no discurso do participantes da pesquisa em menor escala, ou seja, menor de 10, apareceu a educação e interdisciplinaridade.

Gráfico 1. Níveis de ocorrência das barreiras para uma comunicação eficaz entre equipe de trabalho, nas unidades de análise.

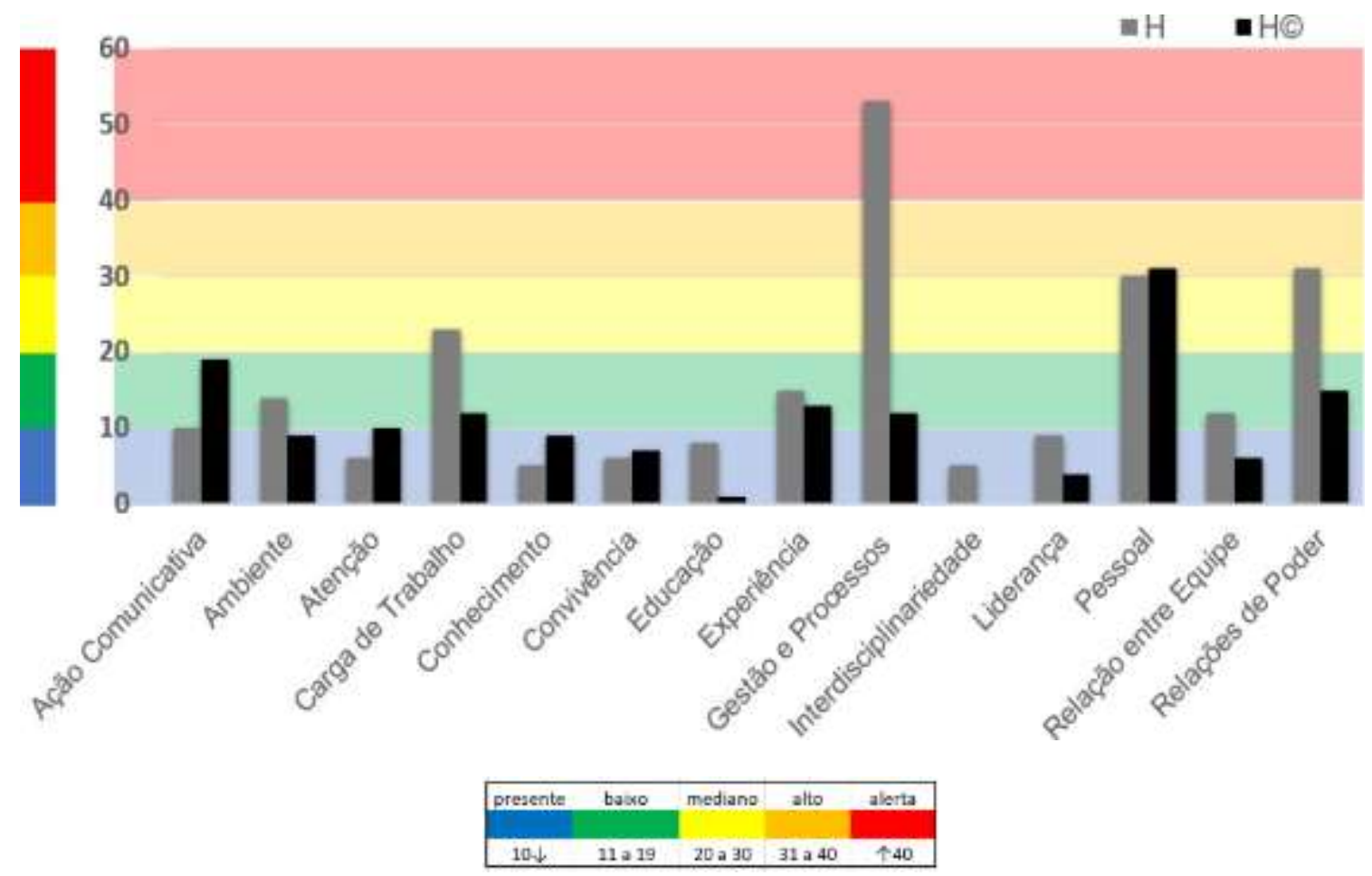

Fonte: Dados da pesquisa. 
Gráfico 2. Níveis de ocorrência das barreiras para uma comunicação eficaz entre equipe de trabalho, nas unidades de análise.

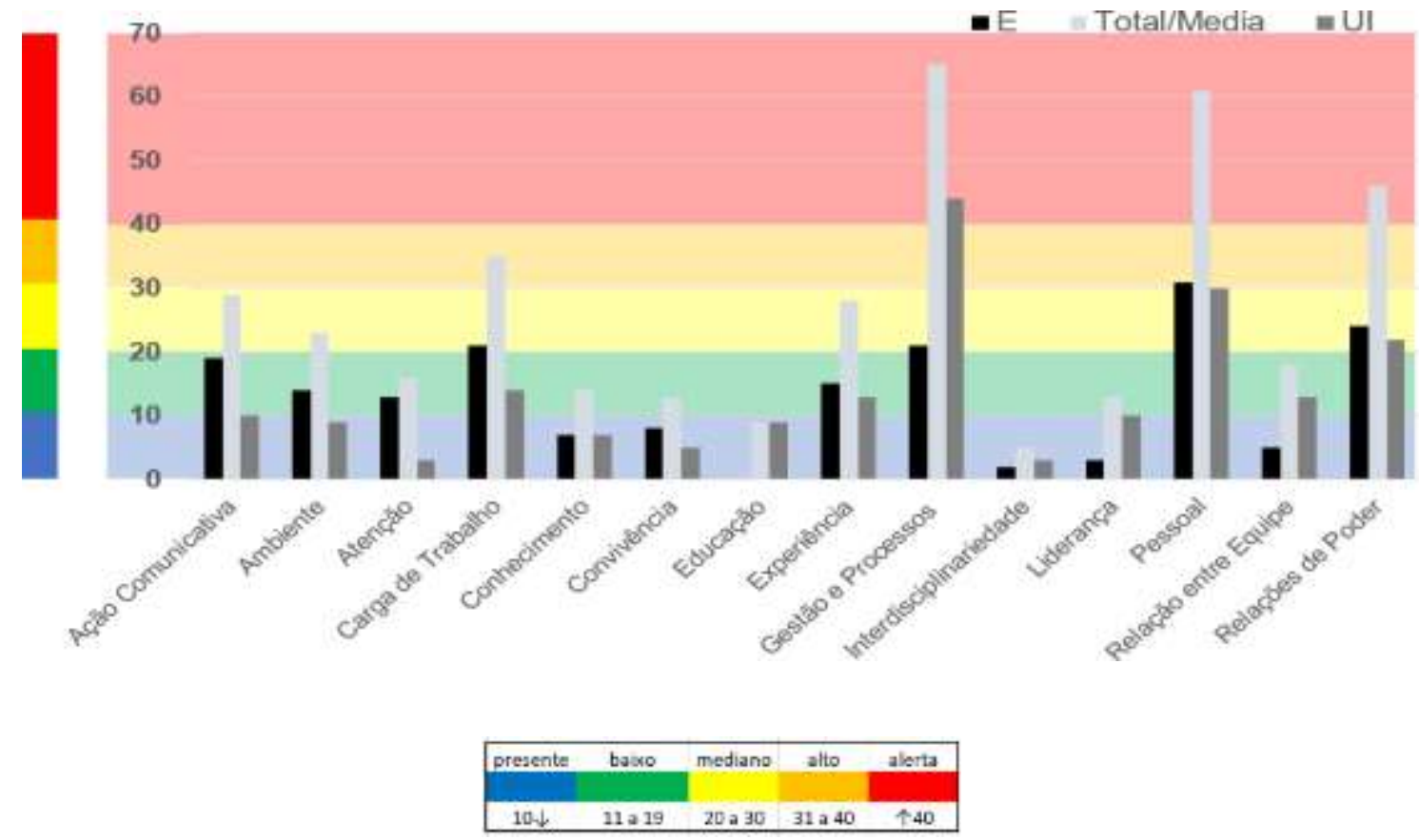

Fonte: Dados da pesquisa.

$\mathrm{Na}$ discussão e análise comparativa deu-se ênfase às categorias em níveis de maior disparidade e frequência. Desta forma, inicia-se pela categoria mais importante: Gestão e processos, destacando-se pela diferença entre as unidades de análise e pela frequência elevada de aparição.

Gestão e processos: marcada pela diversidade de fatores, a instituição de protocolos e rotinas para melhorar a segurança do paciente, foi um dos maiores desafios para efetivação do cuidado seguro, conforme o relato de P13: “ sempre foi um problema do hospital, um problema de protocolo... tem no sistema, no repositório de documentos [...] mas tu aplicar é muito difícil, tu pode tentar, mas vai vir alguém te cobrar que tu está fazendo fora, porque naquele momento a emergência exige, essa semana a emergência exige que seja assim, vem o controle de infecção e diz que isso não é viável, a farmácia... tu está ali no meio, não existe um consenso, é bem frustrante”.

A incongruência entre as exigências organizacionais e a demanda existente, aspectos culturais entre público e privado, burocratização, déficits nos treinamentos, localização de profissionais, problemas estruturais e materiais, somaram-se às dificuldades enfrentadas:

As garantias do emprego, faz com que o pessoal relaxe um pouco (P24);

O nosso trabalho é cada vez mais demandas burocráticas (P22);

Nosso prontuário não é todo eletrônico (P23);

Quase nunca a gente faz treinamentos sobre rotinas básicas de enfermagem...uma coisa importante é tu lançar estratégia de educação continuada sobre as equipes (P22);

Os residentes não têm o mesmo treinamento, que nós temos (P20).

As falhas de comunicação, inclusive a troca de informação técnica entre a equipe a respeito da assistência ao paciente, representaram outra forte barreira a ser superada, motivada por uma série de fatores. A passagem de plantão foi considerada 
um momento importante, especialmente quando ocorre o round, por propiciar o diálogo entre equipe multidisciplinar, em contrapartida, também descrito um método confuso e banalizado:

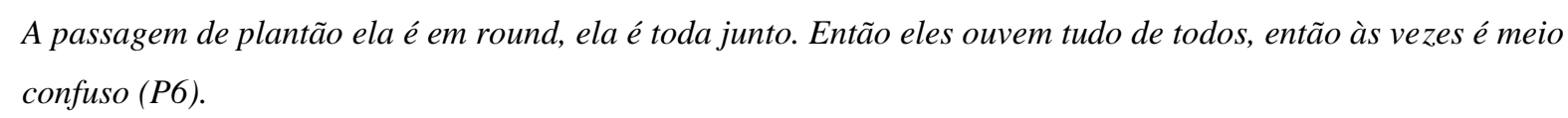

É um momento extremamente importante para a enfermagem, para a equipe multidisciplinar, e a passagem de plantão ela tem perdido norte [...] ela tem que ter o tempo e ela tem que ser sucinta, tem que ser clara e objetiva. Na assistência direta eu acho que a gente perdeu muito em qualidade nesse processo (P23).

O sistema informatizado de prescrição médica produziu alguns vieses, pois o recurso de copiar e colar prescrições anteriores acabou gerando erros e dúvidas, o que aparece no relato de P15: "se o médico do paciente não está ali, a gente bipa o plantão e questiona eles, isto acontece assim muito... aqueles copiam e colam da prescrição”.

A transferência de cuidados entre unidades assistenciais, pode ser comprometida dependendo da percepção do enfermeiro. Isso ficou evidente no caso relatado de transferência de paciente instável por P23: "não sei sinceramente se este paciente foi avaliado antes de ser transferido".

Com a mesma importância e largamente citado estiveram as questões de gerenciamento de pessoas e liderança, e variou desde questões culturais de valorização profissional dentro da instituição até o desenvolvimento de liderança, de acordo com os relatos de P19: "no serviço público não é o conhecimento que te faz se empoderar e sim o tempo dentro da instituição [...] a formação de lideranças e a gestão profissional é o nosso maior desafio".

De forma peculiar a instabilidade política afetou uma das instituições, impactando não só na assistência ao paciente, mas na gestão de pessoas, segundo a fala de P19: “é uma instituição que ela oscila conforme o panorama político, então impacta na não continuidade do processo e na falta de gestão profissional". Para este mesmo profissional, a falta de apoio técnico de áreas fundamentais para a segurança do paciente, tais como: o controle de infecção e o gerenciamento de risco, somam-se às questões de gestão: "a gente tem falta de apoio técnico de áreas como a gestão de risco controle de infecção".

De forma geral, o nível de classificação em alerta, também foi detectado em outras duas categorias: pessoal e relações de poder.

Pessoal: De um lado as expectativas negativas baseadas em atitudes prévias não desejáveis de um indivíduo, do outro, as expectativas positivas geradas em torno de uma pessoa conhecida entre a equipe, ambas foram capazes de perturbar as relações interpessoais:

Seja por uma indisposição prévia como médico... ah, eu sei que o fulano é chato, não vou ligar para ele (P5).

Um médico bem conceituado... justo essa não questionou por que o médico prescreveu é lei (P8).

Pessoas que por um lado tu pensa que tu conhece e daqui a pouco desnorteia tudo (P13).

Divergência de opiniões e interesses, sentimentos como medo e vergonha, personalidade e história de vida, também foram capazes de perturbar os relacionamentos:

É da personalidade da pessoa, da pessoa falar, e isso envolve a timidez, o medo de falar (P1).

Aquele que é muito novo, ele vai ter mais dúvidas... e vai acabar errando por medo de represália ou alguma coisa $(P 2)$. 
Caráter vem de casa, de berço (P2).

Uns são mais lentos outros são mais ativos (P15).

O profissional que é mais desatento... tem muitos problemas lá fora (P15).

Há profissionais e profissionais (P18).

Vai da índole da pessoa (P18).

De forma peculiar a falta de humildade marcou alguns discursos, entre eles o de P10: "ter a humildade de questionamento... hoje em dia nas pessoas que acham que já sabem tudo, principalmente no início da carreira profissional".

Relações de poder: sob a hipótese de superioridade, seja pela hierarquia institucional, cultura médico centrada, egocentrismo e subserviência da enfermagem, a capacidade de questionamento frente a dúvidas e atos inseguros foi prejudicada, constituindo uma forte barreira ao diálogo aberto:

Cultural entre a medicina de enfermagem e que é um desgaste muito grande para a gente conseguir conquistar nosso espaço (P17).

Porque eu vi que o jeito dele, era tipo: eu em cima e tu embaixo, hierarquia (P21).

O hospital tem uma relação hierárquica, então aquele médico veterano e eu sou só um enfermeiro (P22).

Não me sinto como mesmo tipo de liberdade para chegar e contestar o médico do que o técnico (P24).

O estrelismo... e tinha residentes para quem o médico queria ensinar (P24).

A relação hierárquica esteve presente em outros relacionamentos e categorias profissionais, inclusive entre enfermeiro e técnico de enfermagem:

É mais fácil no momento que uma ordem vem um pedido médico, do que uma liderança ou alguma coordenação (P2). Os técnicos de enfermagem preferem tirar muito a dúvida entre si do que com o enfermeiro, não sei se por medo (P22).

Em nível alto, evidenciou-se apenas a categoria carga de trabalho.

Carga de trabalho: relacionada à quantidade e simultaneidade de informação, pressão de tempo, número de pacientes e sua gravidade, equipes grandes, dimensionamento pessoal inadequado, multiempregos, horas extras:

O funcionário admitiu que estava cansado... ele estava fazendo hora extra (P9).

Quando eu tinha uma equipe menor... era muito mais simples (P13).

A unidade muito cheia, poucos funcionários... e pacientes muito dependentes... muitos pacientes usando bomba de infusão (P15).

Isto colaborou para déficits de atenção, realização de tarefas sem o senso crítico (citado pelos participantes como automatização de tarefas), e conflitos entre equipe:

A equipe médica tem sobrecarga de trabalho, chegam momentos que eles ligam o botão automático (P13). 
Os residentes também estão atolados de trabalho e querem resolveras coisas deles... então eles criam esse distanciamento com a enfermagem (P22).

A gente se tornou meio mecanicista... porque a demanda ela é grande (P23).

No nível mediano identificou-se categorias tais quais: ação comunicativa, experiência e ambiente.

Ação comunicativa: as falhas geradas pela falta de comunicação no sentido de construção do diálogo aberto entre equipe sem julgamento de valor, com fins de compartilhar informações importantes para continuidade da assistência, envolveu os processos de escuta, problemas de elaboração e emissão da mensagem pelo locutor, a falta de compreensão pelo receptor e a veracidade da informação:

O processo de revalidação da dor, cada um faz de um jeito. Tu entende que quando eu te passo, não é o que tu entende? (P3).

Se adequar com cada pessoa, criar habilidades de comunicação para cada nível de compreensão (P18).

Nem todas têm a mesma forma de compreensão e a forma de se expressar (P20).

Transmitir as informações corretamente, verdadeiras, embasadas (P23).

A utilização de ferramentas como readback e comunicação fechada para uma comunicação mais efetiva também foram consideradas fundamentais para a segurança do paciente, especialmente na unidade de análise em que a cultura de segurança é amplamente trabalhada e instituída, conforme destacou P5: "a gente trabalha com readback, para uma comunicação efetiva, fechada".

Experiência: incluiu a falta de experiência profissional e a falta de senso crítico associada à autoconfiança, a realização de tarefas habituais e até mesmo as falhas de processos recorrentes, como os erros de prescrição médica. De acordo com alguns profissionais:

Outros acontecimentos podem acabar permitindo que o profissional faça esse tipo de dedução... o médico só copiou a prescrição anterior e não suspendeu... pode acabar levando a pessoa a ter essa liberdade (P11).

A gente usa todas as medicações, já sabe o que vai acontecer... não se vê a necessidade, tu naquela loucura, ir lá na farmacêutica (P13).

O técnico que é mais antigo tem aqueles vícios, manias (P13).

Ambiente: o número elevado de pessoal e intensa circulação tornaram o ambiente vulnerável e propício ao erro:

Ambiente hospitalar, a gente tem que trabalhar com muita segurança (P5).

Muitos residentes, então, têm essa dificuldade de ver quem que é de quem (P21).

Aspectos inerentes às subunidades de pesquisa foram evidenciados por participantes da pesquisa:

Se tratando como a gente trabalha em emergência eu acho é aquela coisa de porta aberta (P2).

Então acaba ficando bem próximo do outro, porque a demanda precisa (P14).

Isso é muito forte na unidade de internação... outros setores, uma relação de continuidade, é mais tranquilo (P22). 
Ele escolhe fazer assim (referente a não utilização de EPIs pelos profissionais da saúde), porque... acha inútil naquele ambiente da emergência (P24).

Na sequência, seguem categorias encaixadas no nível baixo, mesmo em menor frequência, tratando-se de possíveis danos a pacientes, devem ser devidamente consideradas.

Relações entre equipe: representou questões peculiares de relacionamento entre as equipes, em especial na enfermagem, um aspecto cultural de concorrência, segundo P22: "relação entre enfermeiros que às vezes parece uma relação muito mais de competição do que de coleguismo [...] não vai deixar para chegar na passagem de plantão com 14 técnicos de enfermagem e falar [...] talvez o enfermeiro da noite vá reclamar”.

Preocupações com relacionamentos e a própria reputação também permearam as equipes:

Vai pensar estou no contrato ou de repente vão pensar que eu não sei tal coisa (P2).

Depende, eu não vou expor a pessoa, se eu vejo que vai dar um baita de um erro (P9).

As equipes de saúde ainda trabalham de uma forma ainda muito a proteger os seus. Então esse corporativismo, que não é só médico, e talvez seja muito maior na enfermagem, ele acaba prejudicando (P19).

Atenção: indicou o estado de vigília necessário para o exercício da profissão e as ações automáticas, ditas livres do pensamento crítico em determinadas situações:

O técnico (técnico de enfermagem) não tem o pensamento crítico em relação àquela medicação, em relação àquilo que está fazendo, e faz no automático (P12).

A própria rotina do serviço e o hábito, o costume: tá prescrito vou fazer (P16).

A minha maior dificuldade é a falta de atenção, eles (técnico de enfermagem) acham que fazendo no automático é sempre igual, e, muitas vezes, tem que ter atenção no ler e interpretar (P20).

Ele (médico plantonista) está dando esses apagões, se focar muito no procedimento, não enxergar a equipe, não enxerga o paciente (P24).

Conhecimento: caracterizado pela falta de conhecimento técnico-científico, destacado por alguns participantes da pesquisa como parte essencial para prestação de cuidados e na gestão:

Falta de conhecimento do profissional na área da emergência (P3).

O que pesa é a falta de conhecimento técnico... saber gestão, liderança (P19).

Liderança: competências de liderança influenciaram na condução do trabalho e gerenciamento de pessoas, exemplificado com atitudes e preocupações do enfermeiro:

A equipe médica está preocupada em tratar o paciente, eu estou preocupada em tratar o paciente, proteger a minha equipe e seguir a regra da instituição $(P 1)$.

Tem que se pensar em todo contexto... onde não se pense em um assédio moral (P10).

A funcionária cheia de coisas para fazer, eu vou lá e faço (P15).

Eu gostaria de: eu, como enfermeira, de treinar o meu técnico novo (P23). 
Contudo, as demandas já mencionadas, relacionadas a carga de trabalho, muitas vezes burocráticas, ou mesmo por uma questão de postura do profissional junto à equipe, acabou gerando um certo distanciamento entre enfermeiro e técnico de enfermagem, como dito por P22: “como a gente acompanha o trabalho dos técnicos de enfermagem: se é no sentido de fiscalização [...] ou se é no sentido de integração... a forma como vou liderar uma equipe [...] essa postura do enfermeiro, afastada da assistência, pode prejudicar a comunicação dentro da equipe de enfermagem, e os técnicos de enfermagem acabam vendo o enfermeiro quase como um opositor do que alguém que faz parte da sua equipe".

Convivência: representaram os fatores atribuídos pelos participantes da pesquisa, dependentes do tempo que a equipe trabalha junto em um determinado ambiente, entre eles a familiaridade, confiança, intimidade e liberdade. Para fins desta categoria tais fatores afetaram diretamente o relacionamento entre equipe, sendo citados como causa das falhas de comunicação. A confiança entre a equipe de trabalho pode trazer alguns vieses, quando compromete atos seguros, como a dupla conferência, muito comum no processo de medicação, na medida em que não é executada ou não há atenção devida para tal processo crítico, conforme verificado na fala de P10: “devido à enfermeira ter confiado, e não ter prestado atenção no questionamento".

Presente com uma frequência menor de 10 citações, a educação e a interdisciplinaridade emergiram nas falas dos enfermeiros.

Interdisciplinaridade: a falta de apoio interdisciplinar e intersetorialidade esteve presente em alguns relatos:

A gente tem falta de apoio técnico (P19).

Qualquer categoria profissional tem poder para delegar uma regra em cima da enfermagem..., do contrário, quando a enfermagem vai se posicionar com as outras profissões é uma relação muito de pedido (P22).

Com as outras categorias, eu não tenho como ter uma ação mais efetiva em relação ao que eles fazem (P24).

Educação: déficits na formação em enfermagem, diferença entre níveis de aprendizado e instituições de ensino superior impactaram na falta de interdisciplinaridade e trabalho colaborativo entre as equipes:

Eles ainda são de um modelo de formação extremamente hierárquico verticalizado (P19).

A formação para enfermagem é muito importante, tem colegas que qualquer coisa que o médico prescreveu eles vão ir lá e vão fazer... não desenvolveram essa habilidade para conseguir se posicionar (P22).

\section{Discussão}

A característica principal da amostra se deu por profissionais experientes e na faixa etária acima de 30 anos. Perfis de idade e tempo de experiência, demonstraram relevância em outro estudo, com alunos de pós-graduação em enfermagem na China, quando atitudes negativas em relação à cultura de segurança variaram de acordo com a idade e experiência (Kong et al., 2019).

Em nossa pesquisa, a comunicação interprofissional sofreu influência de diversos fatores que agiram como barreiras para sua efetivação. $\mathrm{O}$ aspecto multifatorial dos eventos adversos evidenciados em nosso estudo, se aproximou de um outro, no qual revelou os eventos adversos raramente relacionados a um único fator isolado (20,71\%), cuja maioria dos casos, envolveu múltiplos fatores contributivos (79,29\%) (Mushtaq et al., 2018).

De acordo com nossos objetivos, identificou-se os fatores intrínsecos às relações humanas e organizacionais capazes de influenciar a comunicação interpessoal, gerando incidentes a pacientes hospitalizados em diferentes contextos. Porém, 
mesmo se tratando de uma análise comparativa, na discussão foi dada ênfase às categorias e níveis de maior frequência, devido à complexidade de análise em contextos tão diferentes. Dificuldades semelhantes foram reconhecidas no estudo de Gillespie et al. (2017), diante da tentativa de especular se as diferenças no desempenho de habilidades não técnicas de equipes de cirurgia, podem ser atribuídas a hospitais, especialidades, equipes cirúrgicas.

Entre as barreiras para uma comunicação eficaz entre equipe de trabalho, as categorias mencionadas mais de 60 vezes foram: gestão e processos, e pessoal. Na primeira houve uma diferença díspar entre as unidades de análise $(\mathrm{H} n=53, \mathrm{H} \odot \mathrm{n}=12)$, enquanto nas subunidades não tão expressiva ( $\mathrm{n}=21$, UI $\mathrm{n}=44)$. A disparidade envolveu questões pontuais da instituição pública de análise: turnover e dimensionamento de pessoal; desenvolvimento de lideranças; criação, implantação e acompanhamento de protocolos, fluxos e rotinas; falhas de informação tanto assistencial (registros, transferência de cuidados e prescrição médica) quanto organizacional (ferramentas, gestão, acesso e burocratização da comunicação); processos assistenciais frágeis pela dependência exclusiva de barreiras humanas; déficit de estratégia de treinamentos; valorização profissional; problemas de gerenciamento de profissionais e riscos; questões de inadequação estrutural e material.

Muitos desses achados assemelham-se ao estudo descritivo com 1.092 enfermeiros de hospital público e privado na Turquia, com o objetivo de determinar os erros praticados por enfermeiros. As razões dos erros estavam relacionadas a motivos pessoais, organizacionais e outros profissionais; a atribuição das falhas ao hospital foram causadas por incerteza sobre tarefas, autorizações e responsabilidades, ambientes negativos, instabilidade das unidades de trabalho das enfermeiras, a falta de sistema que poderia prevenir erros médicos; limitações de recursos devido a razões econômicas; a falta e o não cumprimento de protocolos e procedimentos; falha na promoção de treinamentos em serviço; sistema de comunicação insuficiente/desatualizado (Kahriman \& Öztürk, 2016).

A realidade exposta em nossa pesquisa, quanto a mudanças de equipe, registros e treinamentos, também dificultam a comunicação interpessoal na opinião de residentes de emergência (Olde Bekkink et al., 2018). Outros autores evidenciaram os problemas com a documentação do prontuário do paciente entre as causas de eventos adversos de medicação (Manias et al., 2019)

A literatura reforça a importância disso, ao passo que a transferência de informações e compreensão compartilhadas são umas das principais causas de falha de comunicação entre enfermeiros e médicos extraídos de relatórios de incidentes (Elizabeth Umberfield, Amir A Ghaferi, Sarah L Krein, 2019). Não se detendo apenas a relação médico enfermeiro, problemas de omissão e atrasos na assistência de enfermagem são impactados pela transferência de informação deficiente (Albsoul et al., 2019).

Ainda, as questões estruturais, dimensionamento pessoal, comunicação, protocolos, registros e intersetorialidade, identificadas neste estudo foram proporcionais aos encontrados em Butão. Entre eles a falta de pessoal, infraestrutura, falta de políticas, diretrizes e protocolos, comunicação e colaboração deficientes, contribuem com as preocupações dos profissionais da saúde sobre a segurança de paciente. A falta de comunicação, a documentação clara do atendimento prestado, a baixa colaboração entre departamentos do hospital e de trabalho em equipe foram consideradas problemáticas (Pelzang \& Hutchinson, 2018).

De forma complementar, uma análise sistemática de dados no Reino Unido, revelou não só a falta de adesão às políticas e procedimentos, bem como a sua inexistência, como contribuintes em incidentes cirúrgicos (Mushtaq et al., 2018). Isso demonstra que a criação e publicação de protocolos e rotinas não são o suficiente para execução dos mesmos e engajamento da equipe, conforme mencionado por alguns enfermeiros nessa pesquisa, talvez o maior desafio esteja na sua implantação e controle. 
A questão pessoal funcionou como uma barreira de diversas formas, as expectativas pessoais com relação a um determinado profissional, sejam elas positivas ou negativas, levou a tomada de decisão errônea, equivocada, livre de pensamento crítico. Sobre este aspecto, cabe a fundamentação segundo a teoria da ação comunicativa de Jürgen Habermas (1997), na qual, a certeza que acompanha a crença em uma pessoa apoia nas experiências passadas e não exclui o ato de acreditar na possibilidade de equívoco. A certeza não sensível, que se associa aos atos de compreensão, está imunizada contra a possibilidade de descobrir um equívoco posteriormente.

Características individuais de personalidade, cognitivas e sentimentais evidenciaram-se, tornando ímpar o processo de comunicação entre equipe, no entanto, nem todas foram encontradas na literatura recente sobre o assunto, geralmente abordadas de maneira geral, a exemplo da insegurança, emoções pessoais, medo (Olde Bekkink et al., 2018), personalidade, senso de responsabilidade e profissionalismo (Green et al., 2017). Em particular, o medo aparece bem descrito na literatura, tanto na ocasião do relato depois da ocorrência dos eventos adversos (Kahriman \& Öztürk, 2016), quanto antes que aconteçam (Campbell et al., 2019).

Referente ao comportamento de enfermeiros, não diferente de nossos achados, erros são cometidos e colocam a vida do paciente em risco por razões pessoais, tais quais: a irresponsabilidade, a apatia, o desinteresse pessoal, o desgosto profissional e a falta de auto confiança (Kahriman \& Öztürk, 2016).

Atitudes complacentes de criação de atalhos e descuido dos profissionais da saúde, que não aplicam conhecimento, apesar de detê-lo (Pelzang \& Hutchinson, 2018), as violações de regras incidentes sobre o desempenho humano (Suliburk et al., 2019), somam-se aos fatores humanos como causa de eventos adversos e se assemelham aos nossos resultados nas categorias pessoal e atenção, em contrapartida, a falta de humildade, não evidenciou-se em outras pesquisas.

Ainda que em uma frequência menor de citação quando comparado às categorias gestão e processos e a pessoal, a relações de poder também atingiu nível de alerta. Portanto, assim como em nossa pesquisa, a hierarquia tem forte influência no comportamento profissional (Campbell et al., 2019; Pattni et al., 2019), isso se deve a interesses concorrentes (Duffourc, 2018), a intenção de defender posições e territórios (Campbell et al., 2019), educação e sexo também podem afetar a hierarquia de uma equipe (Green et al., 2017).

Neste contexto se inseriu a relação médico-enfermeiro, cuja aproximação à literatura se confirmou, sendo atribuída a causa de erros na assistência de enfermagem, diante de atitudes de fato consumado, onde os questionamentos não são aceitos pelos médicos (Kahriman \& Öztürk, 2016). Uma análise baseada em equipe, para identificar seus papéis nas metas de comunicação do cuidado no fim de vida, revelou compreensões implícitas das estruturas hierárquicas existentes na saúde e construções históricas sociais do enfermeiro-médico (Strachan et al., 2018).

Paralelo a isso, estão as referências dos enfermeiros, em nossa pesquisa, ao associarem a hierarquia em saúde à cultura médico centrada, e a formação de ambos os profissionais. Foronda et al. (2016), justificam a probabilidade de falhas na comunicação estabelecida nesta relação com diferentes abordagens, tais como: a perspectiva histórica dos enfermeiros num papel subserviente aos médicos; a enfermagem com uma visão holística, orientada para sistemas e rica em inteligência emocional, enquanto os médicos são treinados para uma avaliação objetiva e estruturada no atendimento ao paciente, ou seja, uma natureza filosófica diferente entre as disciplinas.

Mesmo diante desta dificuldade, experiências práticas e entendimentos de médicos sobre o valor dos enfermeiros nas discussões sobre metas do cuidado de pacientes gravemente enfermos, influenciou a inclusão intencional destes profissionais nessas discussões (Strachan et al., 2018). Na prática, em nosso estudo, o reconhecimento do papel do enfermeiro pelo médico ainda demanda muito desgaste emocional conforme relatado por P17 na categoria relações de poder. 
Contudo, os gradientes hierárquicos existem em vários níveis de relacionamento, e não apenas entre médicos e enfermeiros (Olde Bekkink et al., 2018). Nossos achados corroboram com os resultados referentes à barreira de relações de poder, presente inclusive na relação técnico de enfermagem e enfermeiro, identificada no relato e nas inferências dos participantes da pesquisa. Esta última, de forma peculiar, não foi evidenciada na literatura nacional nem na internacional, provavelmente pela composição peculiar da categoria no Brasil (Dec. no 94.406, 1987).

Sob a ótica da análise comparativa entre H e H@ das relações de poder, o primeiro apresentou nível alto e o segundo, nível baixo. Apesar de alto, os enfermeiros do $\mathrm{H}$ não se sentiam inferiorizados, mas relataram dificuldades na prática diária para conquista de reconhecimento, já no $\mathrm{H} \odot$, muitos se sentiam seguros e motivados devido o respaldo institucional, e principalmente pela presença de protocolos e rotinas bem instituídos. Quanto as subunidades, não houve diferença nos níveis, ambos, mediano.

Outras quatro categorias (carga de trabalho, ação comunicativa, experiência e ambiente), com frequência geral mediana de citações, demonstraram as dificuldades vividas pela equipe no estabelecimento das relações e na prestação de cuidado, efetivadas e mediadas pela comunicação.

A carga de trabalho se mostrou multifacetada e atingiu um nível alto na frequência geral de citação entre os enfermeiros, diante da análise coocorrência, o $\mathrm{H}$ e a $\mathrm{E}$ tiveram maior representatividade. O diferencial se tornou explícito quando as falas convergiam para dificuldade de troca de informação de forma segura e efetiva diante do aumento da demanda no setor da emergência, sendo considerado uma característica do setor. Esta instabilidade, resultou em sobrecarga e piora no desempenho sob pressão de tempo da equipe, desequilíbrio entre quantitativo de pessoal e pacientes, além dos efeitos mentais e físicos nestes profissionais.

A sobrecarga de trabalho entre enfermeiros, é igualmente evidenciada em diversas pesquisas, com potencial para aumento dos erros (Hammoudi et al., 2018; Kong et al., 2019). Entre os problemas evidenciados encontram-se cansaço, fadiga, número limitado de profissionais (Kahriman \& Öztürk, 2016), concorrência de múltiplas prioridades (Manias et al., 2019), estes fatores também compuseram a categoria carga de trabalho nesta pesquisa.

Em contribuição ao aumento da carga de trabalho, referido pelos participantes da pesquisa enquanto referência para equipe, foram as demandas extras diante das pressões do sistema administrativo e assuntos não clínicos. Em consonância a isso, um estudo examinou as tendências de erros de medicação, em que médicos e farmacêuticos tiveram chances reduzidas de cometerem falhas em comparação com enfermeiros, a diferença entre as disciplinas se deu na frequência de interrupções e distrações no qual os enfermeiros são submetidos (Manias et al., 2019). As interrupções no fluxo de trabalho são responsáveis por atrasos e omissões na assistência em enfermagem (Albsoul et al., 2019).

A categoria médica também foi reconhecida quanto às demandas excessivas no ambiente de trabalho pelos participantes da pesquisa, impactando diretamente a segurança do paciente e desempenho da equipe. Dados revelados por outros autores, identificaram os efeitos negativos do esgotamento médico, para tanto, incluem-se o aumento de erros durante a assistência (Dewa et al., 2017). O esgotamento médico, está associado a motivadores de nível organizacional, individual e cultural (Grow et al., 2019). Apenas um exemplo disso, o aumento significativo de erros durante a prescrição médica, foi atribuído a multiplicidade de tarefas (Westbrook et al., 2018).

$\mathrm{O}$ fator ambiental, principalmente no $\mathrm{H}$ e na $\mathrm{E}$, dificultou a comunicação entre a equipe, ambos têm características peculiares, como alta demanda, e a última ainda administra as flutuações da carga de trabalho, incluindo instabilidade de paciente, inerentes ao ambiente.

As oscilações e outras características específicas do ambiente, particularmente da unidade de análise E, se aproximaram dos resultados de uma pesquisa realizada em unidade de cuidados especializados, ao demonstrarem que a carga 
de tempo dedicado a execução de tarefas rotineiras específicas, podem contribuir diretamente com erros. Interrupções também são reconhecidas como parte da natureza de unidades especializadas (Heneka et al., 2019). Inclusive, as interrupções colaboram significativamente, com o aumento de taxa de erro durante a prescrição médica (Westbrook et al., 2018).

Fez parte do ambiente questões de Iluminação, ruídos, barulho e locais agitados. Em conformidade a isso, uma pesquisa em hospital pediátrico Australiano, detectou a estrutura do hospital, associada às chances reduzidas de erro de medicação (Manias et al., 2019). Uma outra na Turquia, apontou os motivos de erros assistenciais em saúde, no qual, entre as razões relacionadas ao hospital, encontrava-se um ambiente físico negativo (Kahriman \& Öztürk, 2016). Para tanto, os desafios ambientais vivenciados durante cirurgias cardíacas, aumentam a complexidade do caso, insere-se neste contexto, os níveis de ruídos atribuídos às comunicações da própria equipe e à tecnologia (Gillespie et al., 2017).

$\mathrm{O}$ volume de pessoas que circulam no hospital, em especial os residentes médicos, ainda mais no caso do $\mathrm{H}$, um hospital escola, tornou o ambiente conturbado, complicando muitas vezes a identificação de profissionais entre eles. Isso também foi revelado por profissionais de emergência, onde o ambiente clínico de alta acuidade e a troca constante nas equipes de saúde, tornou-se uma barreira à comunicação interprofissional (Olde Bekkink et al., 2018).

A ação comunicativa caracterizada pelo diálogo aberto entre a equipe de trabalho definiu esta categoria, mas, os aspectos relacionados à compreensão da mensagem, mostrou-se um ponto crítico. À luz do nosso estudo Jürgen Habermas (1997), define a ação comunicativa como uma interação simbolicamente mediada, orientada por normas obrigatórias que definem expectativas recíprocas de comportamento, que deve ser reconhecida e entendida pelo menos pelos sujeitos agentes. O erro se produz no plano da identificação do objeto, não no plano da apresentação do mesmo objeto, no qual, um, sempre pode identificar erroneamente. A consciência sobre isso, agregada à cultura de segurança institucional, talvez explique níveis mais elevados da barreira ação comunicativa no H®.

A utilização de técnicas de "read back", comunicação fechada, foram citadas pelos participantes da pesquisa como alternativa diante de informações críticas ao cuidado onde ser objetivo e preciso na expressão da ordem ou da preocupação, assim como ao repetir o dito e/ou escrito, mitigaria os eventos adversos. Porém, esta crença, se contrapõe aos resultados de uma pesquisa que verificou falhas contextuais mais comum de erros de comunicação entre médicos e enfermeiros, nos quais objetivos implícitos ou explícitos da comunicação não estavam claros, em outras palavras, embora a mensagem fosse precisa entre os profissionais, estes não tinham o mesmo entendimento dos objetivos da comunicação (Elizabeth Umberfield, Amir A Ghaferi, Sarah L Krein, 2019). Todavia, faltam estudos que provem a eficácia das técnicas comunicacionais (read back, comunicação fechada) na área da saúde.

Outros vieses discorrem sobre a comunicação racional de cunho técnico. Segundo Jürgen Habermas (1997), a ação instrumental orientada por regras técnicas, descansa em um saber empírico, essas regras implicam em cada prognóstico condicionadas sobre sucessos observáveis, físicos ou sociais. O comportamento racional se orienta por estratégias que se apoiam em um saber analítico. A ação racional realiza determinados fins sob condições dadas; enquanto a ação instrumental organiza meios que são adequados ou inadequados, conforme critérios de controle efetivo da realidade, a ação estratégica só depende da valorização correta de possíveis alternativas de comportamento, que resulta de uma dedução executada com a ajuda de valores e máximas.

Portanto, ainda que a aplicação de técnicas comunicacionais na prática assistencial ajude, outras barreiras se fazem necessárias. Visto que, conforme Suliburk et al. (2019), as deficiências de desempenho humano, são responsáveis por eventos adversos na área cirúrgica, e envolvem falhas na cognição de execução (ou seja, falta de reconhecimento, atenção ou lapso de memória), e o viés cognitivo de planejamento de cuidados ou resolução de problemas. 
A falta de experiência desafia ambientes de alta complexidade como os hospitalares. Erros que colocam a vida do paciente em risco estão associados a falta de experiência dos profissionais (Kahriman \& Öztürk, 2016). O equilíbrio dos níveis de pessoal com base em níveis de competência, habilidade, conhecimento e experiência foi frequentemente percebido por médicos como contribuintes para erros de opioides (Heneka et al., 2019). Estes achados corroboraram igualmente entre as unidades de análise e subunidades em nossa pesquisa.

De forma peculiar, a experiência baseada em vivências passadas, hábitos de profissionais experientes e execução de rotinas habituais, representaram perigo à segurança do paciente. Semelhantemente, uma pesquisa qualitativa que explorou a percepção de médicos sobre erros de opioides, mostrou as particularidades de uma unidade especializada, como o alto volume e dose destes medicamentos, esta foi marcada por diferenças em relação às experiências anteriores dos profissionais. Consequentemente, a carga de tempo dos métodos de entrega de opioides e a complexidade de tarefas rotineiras específicas, contribuíram diretamente para erros destes medicamentos (Heneka et al., 2019).

Relação entre equipe, atenção, conhecimento, convivência e liderança, representaram um nível baixo entre as barreiras citadas pelos enfermeiros, e não tiveram um comportamento diferente entre as unidades e subunidades de análise.

A relação entre equipe, marcada por dificuldades entre turnos na enfermagem, inclusive enfermeiros, diferenças de geração, falta de colaboração, e a preocupação com os relacionamentos, predispôs às práticas de cuidado fragmentadas e desumanizadas, um ambiente de trabalho livre de empatia, e, por vezes, conflituoso, de intimidação.

Sob o contexto da preocupação com os relacionamentos, um referencial teórico explicou justamente como as relações interpessoais em saúde afetam a comunicação, o desempenho da equipe e a segurança do paciente. A partir disto, algumas propostas para as relações interpessoais emergiram: o contexto perceptivo influenciado pelos indivíduos que se relacionam; processo de mudança de comportamento interpessoal ao longo do tempo (Lee \& Doran, 2017). Este mesmo motivo, em um outro estudo, inibiu enfermeiros de não relatarem erros de medicação, diante da crença que seus pares iriam julgar incompetência caso isso ocorresse (Hammoudi et al., 2018). Em reforço à importância dos relacionamentos Levine et al. (2020), descrevem que o aprendizado dos profissionais da saúde é certamente o resultado de suas interações com outras pessoas.

A familiaridade, descrita na categoria convivência em nossa pesquisa, é igualmente percebida na literatura tanto como uma barreira quanto um facilitador. Um experimento randomizado baseado em simulação, verificou e testou comportamentos de fala dos anestesiologistas quando um problema com potencial para danos ao paciente, emana na sala de cirurgia. Os resultados revelaram, entre os obstáculos mais comuns, os estereótipos de membros da equipe, respeito e familiaridade. Curiosamente a familiaridade não foi citada apenas como uma dificuldade, mas também como um facilitador (Raemer et al., 2016).

Em conformidade, enquanto barreira, a falta de familiaridade com determinados procedimentos de enfermagem causou erros de medicação (Albsoul et al., 2019). Este último achado, vem ao encontro da execução de cuidados assistenciais sem o senso crítico, citado por muitos participantes da pesquisa como automatização de tarefas, em função da confiança em pessoas, gerando a reprodução de erros, quebra técnica e violações. E, esteve relacionada a outras categorias além da convivência, também a experiência, ambiente, atenção e a carga de trabalho.

O conhecimento mostrou-se fundamental para a prestação de cuidados seguros, para tanto, não faltam evidências na literatura que confirmem isso (Heneka et al., 2019; Kahriman \& Öztürk, 2016; Manias et al., 2019; Pelzang \& Hutchinson, 2018). Todavia, não se mostrou suficiente para garantir a segurança do paciente, na medida em que, a falta de humildade e autoconfiança apareceram juntamente com esta categoria, cuja associação, resultou em atos inseguros. 
Research, Society and Development, v. 10, n. 7, e10810716218, 2021

(CC BY 4.0) | ISSN 2525-3409 | DOI: http://dx.doi.org/10.33448/rsd-v10i7.16218

Características indesejáveis de um líder, responsabilidade sobre a condução da equipe e dos cuidados assistenciais, tomada de decisão frente as situações tênues entre atos inseguros de profissionais da saúde e a garantia do respeito e preservação da imagem da sua equipe ou indivíduo, tornou ambíguo e desafiador o exercício e o desenvolvimento do enfermeiro enquanto liderança, nesse contexto complexo se formatou a categoria liderança. Mesmo assim, independentemente da existência de tais habilidades e competências, enfermeiros são uma parte essencial na gestão de processos e liderança de equipe. Nesse sentido, Levine et al. (2020), salientam as funções de enfermeiros e gerentes de enfermagem, como promotores de mudanças na cultura e no clima da organização, ao passo que, este último está relacionado à intenção de falar dos funcionários, sobre erros assistenciais.

Como visto, as funções profissionais do enfermeiro vão além das assistenciais, em nossa pesquisa, eles atuam como corretores de informações, defensores e apoiadores para pacientes e familiares, mostrando-se uma referência na equipe de saúde. Em contrapartida, Strachan et al. (2018), trazem que, nem sempre a comunicação do cuidado pelo enfermeiro é reconhecida, pois surge espontaneamente durante o cuidado e ocorre de forma assíncrona e fora dos processos de tomada de decisão mais frequentemente pesquisados, testados, formais e planejados.

A valorização à manifestação das preocupações da equipe sobre segurança e qualidade assistencial também foi uma preocupação entre enfermeiros enquanto líderes de equipe, entretanto, um estudo qualitativo em hospitais, identificou os próprios mecanismos destinados a facilitar a comunicação, inibindo a fala dos funcionários, diante disso, estratégias relacionais eram adotadas pelos líderes. Também importante, era a preocupação destes em minimizar a exposição aos riscos de litígios, reclamações de intimidação ou discriminação ou ação sindical (Martin et al., 2018), com esta justificativa, alguns participantes de nossa pesquisa adotaram uma postura de preservação de imagem e prevenção de exposição pessoal, diante de atos inseguros em curso que podiam culminar em danos ao paciente.

A falta de atenção, no presente estudo, embora numa frequência baixa, indicou a automatização de tarefas, ou seja, a realização de tarefas livres do pensamento crítico. Por isso, admite-se que errar é humano, devidamente descrito nas pesquisas relacionadas ao assunto. Uma delas, envolvendo profissionais médicos na Austrália reconheceu que falhas ativas como escorregões, lapsos e erros eram inevitáveis durante o processo de entrega de opioides (Heneka et al., 2019). A outra, identificou as falhas cognitivas entre os contribuintes em incidentes cirúrgico, as frequentemente encontradas são falhas na atenção, memorização, concentração, resolução de problema e tomada de decisão (Mushtaq et al., 2018).

Nesse contexto, ao que pese a complexidade da tarefa, uma pesquisa avaliou a capacidade de memória de trabalho de médicos de emergência, cuja medida foi através de erros de prescrição. A parte mais complexa da tarefa de prescrição é decidir sobre os elementos clínicos, outros, como selecionar abreviações corretas. Portanto, médicos cometeram mais erros clínicos quando interrompidos, mas os erros legais/procedimentais não foram afetados, isso pode ser explicado por diferenças na complexidade da tarefa (Westbrook et al., 2018). Isso explicaria a relação entre automatização de tarefas afetada pelo ambiente e carga de trabalho em nosso trabalho.

O fazer automático, considerando-se, uma falta de abordagem crítica, ao cumprir ordens sem haver questionamentos, foram razões pessoais elencadas por enfermeiras, para erros assistências (Kahriman \& Öztürk, 2016). Este achado ainda parece obscuro, merecendo investigações posteriores, pois outros fatores podem estar associados a isso, assim como mencionado nesta pesquisa.

Em uma frequência inferior a 10 citações, a educação e interdisciplinaridade revelaram-se entre as barreiras. A última indicou, não só a falta de apoio entre disciplinas e setores, mas a autossuficiência da enfermagem em compartilhar suas necessidades e dúvidas. Esse resultado corrobora com a falta de trabalho em equipe e colaboração entre os departamentos e os médicos, considerada particularmente problemática, em três hospitais de Butão (Pelzang \& Hutchinson, 2018). 
Já a educação, inscreveu-se sobre déficits na formação, inclusive contemplado uma abordagem interdisciplinar, além da diversidade de níveis de ensino na equipe assistencial. A repercussão disso nos relatos dos participantes desta pesquisa, se aproximou ao estudo de Bragadóttir \& Kalisch (2018), à medida que diferenças em funções e responsabilidades de enfermeiros, com base na sua formação e treinamento, levou a modelos diversificados quanto à prioridade de elementos do cuidado.

\section{Conclusão}

Esta pesquisa mostrou a diversidade de fatores contribuintes ao erro humano, ao que pesem as relações humanas e organizacionais. Além disso, a análise de coocorrência entre distintos contextos, enriqueceu nossos achados. Para tanto, fornecemos dados valiosos, em níveis de frequência, permitindo o direcionamento e prioridade de ações, a fim de mitigar erros nas instituições de análise e melhorar o ambiente de trabalho.

As diferenças entre as unidades e subunidades de análise foram ressaltadas principalmente pelas barreiras: gestão e processos, relações de poder e pessoal. De forma geral, elas têm dificultado a comunicação entre as equipes de trabalho. Em especial a hierarquia, atingiu diversas categorias profissionais, inclusive a relação técnico de enfermagem e enfermeiro, embora nem sempre reconhecida, mas inferida nos seus discursos.

O fazer automático, livre de pensamento crítico, marcou algumas barreiras à comunicação eficaz, curiosamente esteve relacionado com a confiança, experiência, ambiente, atenção e a carga de trabalho. A contravenção a respeito da confiança e experiência no comportamento de profissionais de saúde em ambientes hospitalares, ainda pouco evidenciada e explorada na literatura, mostra a importância de estudos futuros.

Contudo, a enfermagem, como a categoria que passa mais tempo à frente do cuidado, deve destituir-se da autossuficiência, e até mesmo da confiança condicionada à convivência, e buscar apoio interdisciplinar e intersetorial. Para isso, precisamos superar algumas barreiras relacionadas nesta pesquisa.

\section{Referências}

Agency for Healthcare Research and Quality. (2017). Estimando os custos adicionais de internação hospitalar e mortalidade associados às condições selecionadas do hospital. AHRQ. https://www.ahrq.gov/professionals/quality-patient-safety/pfp/haccost2017.html.]

AHRQ. Agency for Healthcare Research and Quality. (2004). Pesquisa hospitalar sobre cultura de segurança do paciente. Pesquisa Hospitalar Sobre Cultura de Segurança Do Paciente. Rockville: AHRQ. Https://Www.Ahrq.Gov/Sops/Surveys/Hospital/Index.Html.

Albsoul, R., FitzGerald, G., Finucane, J., \& Borkoles, E. (2019). Factors influencing missed nursing care in public hospitals in Australia: An exploratory mixed methods study. International Journal of Health Planning and Management, 34(4), e1820-e1832. https://doi.org/10.1002/hpm.2898

Bardin, L. (2016). Análise de conteúdo (70th ed.).

Bragadóttir, H., \& Kalisch, B. J. (2018). Comparison of reports of missed nursing care: Registered Nurses vs. practical nurses in hospitals. Scandinavian Journal of Caring Sciences, 32(3), 1227-1236. https://doi.org/10.1111/scs.12570

Brasil Ministério da saúde. (2013). Portaria n 529, de01 de abril de 2013, institui o Programa Nacional de segurança do paciente. Brasília(DF): Ministério Da Saúde.

Campbell, A., Sutcliffe, K., Carlstrom, M., Clements, J., Martin, G., Dixon-Woods, M., Aveling, E., Willars, J., Tarrant, C., \& Pronovost, P. (2019). Improving Employee Voice about Transgressive or Disruptive Behavior: A Case Study. Academic Medicine, 94(4). https://doi.org/10.1097/ACM.0000000000002447

Codier, E., \& Codier, D. D. (2005). Make Patients Safer? American Journal of Nursing, 117(7), 58-62.

Cooper, J. (2017). Mixed Methods Analysis of a National Database. Annual Familly Medecine, 25, 455-462.

Dec. no 94.406, de 08. 06. 8. (1987). Regulamenta a Lei no 7.498, de 25 de junho de 1986, que dispõe sobre o exercício da Enfermagem, e dá outras providências. Brasilia/DF. WSHtNC05DAMtRqFL1RiDBSXLj0 http://www.cofen.gov.br/decreto-n-9440687_4173.html?fbclid=IwAR0F7EIRZQ1_D8FxzMg2bIiJffBc2uT8- 
Research, Society and Development, v. 10, n. 7, e10810716218, 2021 (CC BY 4.0) | ISSN 2525-3409 | DOI: http://dx.doi.org/10.33448/rsd-v10i7.16218

Dewa, C. S., Loong, D., Bonato, S., \& Trojanowski, L. (2017). The relationship between physician burnout and quality of healthcare in terms of safety and acceptability: A systematic review. BMJ Open, 7(6). https://doi.org/10.1136/bmjopen-2016-015141

Duffourc, M. N. (2018). Filling voice promotion gaps in healthcare through a comparative analysis of error reporting and learning systems and open communication and disclosure policies in the United States and Germany. American Journal of Law and Medicine, 44(4), 579-605. https://doi.org/10.1177/0098858818821137

Umberfield, E., Ghaferi, A. A., Krein, S. L., \& Manojlovich, M. (2019). Using Incident Reports to Assess Communication Failures and Patient Outcomes. Joint Commission journal on quality and patient safety, 45(6), 406-413. https://doi.org/10.1016/j.jcjq.2019.02.006

Figueiredo, T. W. B., Da Silva, L. A. A., Brusamarello, T., De Oliveira, E. S., Dos Santos, T., \& Pontes, L. (2018). Tipos, Causas E Estratégias De Intervenção Frente a Erros De Medicação: Uma Revisão Integrativa. Revista de Enfermagem e Atenção à Saúde, 7(2), 155-175. https://doi.org/10.18554/reas.v7i2.2494

Foronda, C., MacWilliams, B., \& McArthur, E. (2016). Interprofessional communication in healthcare: An integrative review. Nurse Education in Practice, 19, 36-40. https://doi.org/10.1016/j.nepr.2016.04.005

Gillespie, B., Kang, E., Chaboyer, W., Harbeck, E., Steel, C., \& Fairweather, N. (2017). Correlates of non-technical skills in surgery: A prospective study. BMJ Open, 7(1). https://doi.org/10.1136/bmjopen-2016-014480

Green, B., Oeppen, R. S., Smith, D. W., \& Brennan, P. A. (2017). Challenging hierarchy in healthcare teams - ways to flatten gradients to improve teamwork and patient care. British Journal of Oral and Maxillofacial Surgery, 55(5), 449-453. https://doi.org/10.1016/j.bjoms.2017.02.010

Grow, H. M., McPhillips, H. A., \& Batra, M. (2019). Understanding physician burnout. Current Problems in Pediatric and Adolescent Health Care, 49(11), 18. https://doi.org/10.1016/j.cppeds.2019.100656

Hammoudi, B. M., Ismaile, S., \& Abu Yahya, O. (2018). Factors associated with medication administration errors and why nurses fail to report them. Scandinavian Journal of Caring Sciences, 32(3), 1038-1046. https://doi.org/10.1111/scs.12546

Heneka, N., Bhattarai, P., Shaw, T., Rowett, D., Lapkin, S., \& Phillips, J. L. (2019). Clinicians' perceptions of opioid error-contributing factors in inpatient palliative care services: A qualitative study. Palliative Medicine, 33(4), 430-444. https://doi.org/10.1177/0269216319832799

Habermas, J. (1997). Teoría de la acción comunicativa: complementos y estudios previos (Cátedra S.A (ed.); $3^{\circ}$ ).

Kahriman, İ., \& Öztürk, H. (2016). Evaluating medical errors made by nurses during their diagnosis, treatment and care practices. Journal of Clinical Nursing, 25(19-20), 2884-2894. https://doi.org/10.1111/jocn.13341

Kong, L. N., Zhu, W. F., He, S., Chen, S. Z., Yang, L., Qi, L., \& Peng, X. (2019). Attitudes towards patient safety culture among postgraduate nursing students in China: A cross-sectional study. Nurse Education in Practice, 38(1), 1-6. https://doi.org/10.1016/j.nepr.2019.05.014

Kowalski, S. L., \& Anthony, M. (2017). CE: Nursing's Evolving Role in Patient Safety. The American journal of nursing, 117(2), 34-48. https://doi.org/10.1097/01.NAJ.0000512274.79629.3c

Lee, C. T. S., \& Doran, D. M. (2017). The Role of Interpersonal Relations in Healthcare Team Communication and Patient Safety: A Proposed Model of Interpersonal Process in Teamwork. The Canadian Journal of Nursing Research = Revue Canadienne de Recherche En Sciences Infirmieres, 49(2), 75-93. https://doi.org/10.1177/0844562117699349

Levine, K. J., Carmody, M., \& Silk, K. J. (2020). The influence of organizational culture, climate and commitment on speaking up about medical errors. Journal of Nursing Management, 28(1), 130-138. https://doi.org/10.1111/jonm.12906

Maia, C. S., Freitas, D. R. C. de, Gallo, L. G., \& Araújo, W. N. de. (2018). Notificações de eventos adversos relacionados com a assistência à saúde que levaram a óbitos no Brasil, 2014-2016. Epidemiologia e Servicos de Saude: Revista Do Sistema Unico de Saude Do Brasil, 27(2), e2017320. https://doi.org/10.5123/S1679-49742018000200004

Manias, E., Cranswick, N., Newall, F., Rosenfeld, E., Weiner, C., Williams, A., Wong, I. C. K., Borrott, N., Lai, J., \& Kinney, S. (2019). Medication error trends and effects of person-related, environment-related and communication-related factors on medication errors in a paediatric hospital. Journal of Paediatrics and Child Health, 55(3), 320-326. https://doi.org/10.1111/jpc.14193

Martin, G. P., Aveling, E. L., Campbell, A., Tarrant, C., Pronovost, P. J., Mitchell, I., Dankers, C., Bates, D., \& Dixon-Woods, M. (2018). Making soft intelligence hard: A multi-site qualitative study of challenges relating to voice about safety concerns. BMJ Quality and Safety, 27(9), 710-717. https://doi.org/10.1136/bmjqs-2017-007579

Mushtaq, F., O’Driscoll, C., Smith, F. C. T., Wilkins, D., Kapur, N., \& Lawton, R. (2018). Contributory factors in surgical incidents as delineated by a confidential reporting system. Annals of the Royal College of Surgeons of England, 100(5), 401-405. https://doi.org/10.1308/rcsann.2018.0025

Olde Bekkink, M., Farrell, S. E., \& Takayesu, J. K. (2018). Interprofessional communication in the emergency department: residents' perceptions and implications for medical education. International Journal of Medical Education, 9, 262-270. https://doi.org/10.5116/ijme.5bb5.c111

Pattni, N., Arzola, C., Malavade, A., Varmani, S., Krimus, L., \& Friedman, Z. (2019). Challenging authority and speaking up in the operating room environment: a narrative synthesis. British Journal of Anaesthesia, 122(2), 233-244. https://doi.org/10.1016/j.bja.2018.10.056

Pelzang, R., \& Hutchinson, A. M. (2018). Patient safety issues and concerns in Bhutan's healthcare system: A qualitative exploratory descriptive study. BMJ Open, 8(7). https://doi.org/10.1136/bmjopen-2018-022788 
Research, Society and Development, v. 10, n. 7, e10810716218, 2021

(CC BY 4.0) | ISSN 2525-3409 | DOI: http://dx.doi.org/10.33448/rsd-v10i7.16218

Raemer, D. B., Kolbe, M., Minehart, R. D., Rudolph, J. W., \& Pian-Smith, M. C. M. (2016). Improving anesthesiologists' ability to speak up in the operating room: A randomized controlled experiment of a simulation-based intervention and a qualitative analysis of hurdles and enablers. Academic Medicine, 91(4), 530-539. https://doi.org/10.1097/ACM.0000000000001033

Strachan, P. H., Kryworuchko, J., Nouvet, E., Downar, J., \& You, J. J. (2018). Canadian hospital nurses' roles in communication and decision-making about goals of care: An interpretive description of critical incidents. Applied Nursing Research, 40(December 2017), 26-33. https://doi.org/10.1016/j.apnr.2017.12.014

Suliburk, J. W., Buck, Q. M., Pirko, C. J., Massarweh, N. N., Barshes, N. R., Singh, H., \& Rosengart, T. K. (2019). Analysis of Human Performance Deficiencies Associated With Surgical Adverse Events. JAMA Network Open, 2(7), e198067. https://doi.org/10.1001/jamanetworkopen.2019.8067

Topcu, I., Türkmen, A. S., Sahiner, N. C., Savaser, S., \& Sen, H. (2017). Physicians' and nurses' medical errors associated with communication failures. Journal of the Pakistan Medical Association, 67(4), 600-604.

Westbrook, J. I., Raban, M. Z., Walter, S. R., \& Douglas, H. (2018). Task errors by emergency physicians are associated with interruptions, multitasking, fatigue and working memory capacity: A prospective, direct observation study. BMJ Quality and Safety, 27(8), 655-663. https://doi.org/10.1136/bmjqs-2017007333 